\title{
El trabajo en la trama automotriz argentina: un estudio sobre la segmentación y evolución del empleo a lo largo de una década ${ }^{1}$
}

\author{
Labor in the Argentine automotive network: a study about employment \\ segmentation and evolution throughout a decade*
}

\author{
Ana Miranda \\ amiranda@flacso.org.ar \\ Facultad Latinoamericana de Ciencias Sociales \\ (FLACSO) - CONICET - Universidad de Buenos \\ Aires, Argentina \\ Miguel Alfredo \\ malfredo@flacso.org.ar \\ Facultad Latinoamericana de Ciencias Sociales \\ (FLACSO) - CONICET - Universidad de Buenos \\ Aires, Argentina
}

Recepción: 09 Octubre 2019

Aprobación: 15 Julio 2020

Publicación: 02 Agosto 2020

Cita sugerida: Miranda, A. y Miguel, A. (2020). El trabajo en la trama automotriz argentina: un estudio sobre la segmentación y evolución del empleo a lo largo de una década. Cuestiones de Sociología, 23, e100. https:// doi.org/10.24215/23468904e07
Resumen: El artículo propone aportar al campo de los estudios del trabajo a través de una investigación sobre las ocupaciones de la trama productiva automotriz en el período 2006-2016 en Argentina. Sostiene que existe una interacción entre la segmentación laboral y los entramados industriales de capitales de considerable volumen, lo cual no implica una necesaria e intrínseca lógica de subordinación. La hipótesis central del texto plantea que: a) la trama productiva automotriz cuenta con una organización del trabajo segmentada en donde, b) el movimiento del ciclo económico presenta condiciones diferenciadas al interior de las diversas actividades que la componen. La fuente principal de análisis corresponde a datos cuantitativos. Los segmentos laborales son definidos como segmento primario (vinculados a la producción/fabricación de automotores y autopartes) y el segmento secundario (referente a la venta, reparación y mantenimiento de automotores y sus componentes). Los resultados proponen la expansión de dos fases durante el período en análisis, en donde los segmentos laborales experimentan un comportamiento diferenciado, que afecta la calidad del empleo en el conjunto de la trama.

Palabras clave: Trama productiva automotriz, Segmentos laborales, Empleo.

Abstract: The aim of this paper is to contribute to the field of labor studies with a research about employment in the automotive production network in Argentina from 2006 to 2016. The study demonstrates that there is an interaction between labor segmentation and industrial fabrics of large amount of capital, which does not involve a necessary or intrinsic subordination logic. The main hypothesis suggests that a) the automotive production network has a segmented labor organization where, b) the economic cycle movement presents diverse conditions in the different activities comprising them. Quantitative data is the major source of analysis. The main source of analysis corresponds to quantitative data. The labor segments are defined as the primary segment (linked to the production / manufacture of automobiles and auto parts) and the secondary segment (referring to the sale, repair and maintenance of automobiles and their components). The results 
propose a two-phase expansion during the analyzed period in which the labor segments experience a differentiated behavior, affecting the labor quality of the fabric.

Keywords: Automotive production network, Labor segments, Employment.

\section{INTRODUCCIÓN}

La industria automotriz ocupa un lugar de gran importancia en el desarrollo manufacturero de los países occidentales y representa un tema de estudio significativo en el campo de las investigaciones laborales (De la Garza y Hernández Romo, 2018). Su centralidad ha significado que el proceso productivo se convirtiera en un foco de gran atención, con relación a las nuevas formas organizativas y laborales del capitalismo. Tal es el caso del toyotismo que, sobre finales de los año\$ 0, consolidó nuevas estrategias globales de producción bajo el perfil de "ensamblaje modular", generando una mayor heterogeneidad de la cadena productiva. Esto desencadena a su vez una mayor segmentación del mercado laboral y un aumento en la brecha tecnológica entre los países del Norte y Sur Global (Carbajal Suárez, 2010).

En este marco, y con el objetivo de aportar al campo de los estudios del trabajo, el artículo presenta una investigación sobre las ocupaciones de la industria automotriz en Argentina. Partiendo de la noción de trama productiva y del enfoque de la segmentación laboral, propone una reflexión sobre los vínculos entre condicionantes externos, ciclo económico y configuraciones laborales en el capitalismo periférico. El enfoque es fruto de una interpretación teórica que visibiliza transversalmente los distintos trabajos que conlleva el proceso productivo (Castillo, 2005), que permite un sustento analítico para la visibilización de la amplia gama de puestos y ocupaciones. El recorte temporal abarca el período 2006-2016, un período muy significativo para la industria argentina, al interior del cual se distinguen dos fases según las tendencias económicas.

Como parte de una tradición conceptual de la economía del trabajo en el artículo se describe la segmentación laboral en la trama a partir de las características de los tipos de empresas, las características sociodemográficas y la evolución de los indicadores laborales sobre calidad del empleo. Tanto la evolución de los indicadores como las características seleccionadas se conciben vinculadas a los segmentos de laborales definidos como el segmento primario (vinculado a la producción/fabricación de automotores y autopartes) y el segmento secundario (referente a la venta, reparación y mantenimiento de automotores y sus componentes).

Estos segmentos pertenecientes a los diversos anillos y circuitos de la trama productiva automotriz en Argentina comprenden una amplia gama de actividades. En este sentido los interrogantes que guían al texto son: 1) ¿Qué características asume el ciclo económico de la industria automotriz asociado a la actividad de fabricación de vehículos automotores, remolques y semirremolques en el periodo 2006-2016?; 2) ¿Cuál fue la dinámica que adquirió la organización del trabajo en la trama productiva automotriz dentro de las diversas fases del ciclo económico abordado?; 3) ¿Cómo evolucionó la composición de la demanda laboral y qué características sociodemográficas revistió la oferta de trabajo según los segmentos delimitados?; 4) ¿Cómo evolucionó la calidad del empleo al interior de los diversos segmentos a lo largo del ciclo económico abordado?

El artículo se organiza de la siguiente manera: en un primer apartado se aborda la conceptualización de la trama productiva automotriz. A continuación, se detallan los aspectos metodológicos y las fuentes utilizadas, lo que es precedido por un subítem de caracterización del período 2006-2016. En un cuarto apartado se expone la dinámica de la organización del trabajo en la trama productiva automotriz a partir de los presupuestos teóricos de la segmentación del mercado de trabajo. Por último se analiza la evidencia empírica en base a los tipos de empresas, características sociodemográficas y calidad de empleo en los segmentos de la trama y se presentan un conjunto de reflexiones sobre los hallazgos y la continuidad de la investigación. 


\section{INDUSTRIA AUTOMOTRIZ: ¿SECTOR ECONÓMICO O TRAMA PRODUCTIVA?}

La evolución de los puestos de trabajo y las brechas salariales en América Latina a lo largo del s. XX habilitan a reflexionar sobre cómo los diversos niveles y volúmenes intra e intersectores constriñen y condicionan la estructura ocupacional. Autores inscriptos en el estructuralismo latinoamericano sugieren que las asimetrías de las estructuras periféricas, producto de la absorción diferenciada de los procesos tecnológicos, provocan niveles de productividad diversos entre los sectores y al interior de cada uno. Esta situación deviene en una segmentación del sistema productivo y del mercado de trabajo, retroalimentando las asimetrías de las condiciones tecnológicas, provocando una desigualdad distributiva (Cimoli y Porcile, 2011). El surgir de la localización de nuevos entramados industriales puso en cuestión a esta forma organizativa, señalando que existe una nueva división internacional del trabajo (Frobel, Heinrichs y Kreye, 1978)

La noción de Sur global surge como respuesta a las tendencias globales, que involucran a las relaciones de producción, y una nueva definición de los sujetos. Se constituye en una referencia a las formas de organización social, económica, política y cultural que nuclea regiones, países, grupos sociales e individuos, llevando a repensar cómo se dan los vínculos entre lo local y lo global (Sousa Santos, 2011, p. 35). En este marco, y en consideración ampliada a los estudios focalizados en la actividad industrial "clásica", la notoriedad que toman las tramas productivas tensiona a la noción de producción nacional para determinadas actividades económicas. Así, la integración de elementos tangibles e intangibles pone en tensión las definiciones económicas clásicas de carácter "sectorialistas".

La actividad automotriz en Argentina cuenta con una trayectoria que puede rastrearse a principios del s. XX (Picabea y Thomas, 2015). Según diversos trabajos la expansión de esta se dio a mediados de siglo con la incorporación de capitales en proceso de renovación en los países industrializados, lo cual resultó, junto a una determinada forma de organización del trabajo, en factores objetivos limitantes del desarrollo de la industria (Ianni, 2008; Harari, 2015). Dentro esta línea de factores limitantes objetivos, y entrando en las últimas décadas del s. XX, los movimientos de articulación en el entramado local respondieron a estrategias corporativas de procesos de integración regional por parte de las filiales locales (Guevara, 2011). Este escenario es posible a partir del carácter dependiente de las filiales locales ante las casas matrices, en los procesos de integración regional sectorial (léase MERCOSUR), aún con el marco potencial de la articulación entre los principales socios (Argentina-Brasil), no lograron superar las asimetrías y dependencias estructurales (Treacy, 2018).

En el caso argentino, el período que abarca la finalización del s. XX y el inicio del s. XXI, muestra a las filiales locales de las empresas transnacionales automotrices con un peso estratégico en el entramado local a raíz de su capacidad de influencia en aspectos productivos (con diversos grados de integración con la industria autopartista) como financieros dentro del circuito de venta local (Pinazo, 2015). La particularidad del caso radica en que las estrategias de reconversión productiva impulsadas por las empresas transnacionales automotrices contemplaron una renovación tecnológica en las plataformas ensambladoras y una reorganización de la cadena de proveedores de insumos (Pinazo, 2015). Así, la configuración que la industria automotriz adquirió post-Convertibilidad se ha sustentado en un crecimiento en su nivel de rentabilidad pero no en su nivel de empleo, producto de la lógica empresarial transnacional y en detrimento del papel del Estado y la relación salarial (Santarcángelo y Pinazo, 2009). Estas condiciones marcan que el problema de la industria en Argentina no está dado únicamente por la productividad laboral agregada, sino fundamentalmente por las estrategias productivas de las grandes empresas transnacionales (Pinazo, Dinerstein y Córdoba, 2017).

Atendiendo estas consideraciones, en esta investigación se entiende por trama productiva automotriz al conjunto de actividades económicas y firmas que se nuclean desde la producción (ensamblaje) que se da en las terminales y empresas autopartistas, los servicios de comercialización de los vehículos y componentes, y 
los proveedores de conjuntos y subconjuntos para el ensamblaje, así como los diversos establecimientos que brindan mantenimiento y reparación de los automotores.

En línea con la definición de trama propuesta por Yoguel (2007, p. 146), en la trama productiva automotriz se establecen empresas terminales y proveedoras de primer orden mundial que organizan y gobiernan el entramado. Estas, en contiguo con proveedores locales, brindan conjuntos y subconjuntos en base a la demanda, estableciendo las relaciones de tipo estable que la definición de trama sugiere. El elemento agregado en esta definición de trama productiva automotriz se da con la incorporación de los establecimientos y trabajadores dedicados a la venta, mantenimiento y reparación de los vehículos. Si bien una proporción de estos se encuentran bajo la órbita de las firmas terminales, existe una masa laboral dedicada a la reparación de automotores que no establecen intercambios de conocimientos ni vínculos comerciales bilaterales con las empresas rectoras. De este modo, la incorporación de los trabajadores dedicados a la reparación permite abordar un segmento laboral diferenciado de las grandes firmas y terminales, y que sin duda da respuesta a un parque automotor existente y en funcionamiento.

\section{La trama aUtomotriz en Argentina en el Período 2006-2016}

\section{1. Aspectos metodológicos}

A lo largo de la investigación se trabajó con distintas fuentes de información secundaria. En primer lugar, y con el objetivo de tener una visión general de la trama, se abordaron los indicadores elaborados por el Observatorio de Empleo y Dinámica Empresarial (OEDE) del Ministerio de Trabajo, Empleo y Seguridad Social de la Nación, en base al Sistema Integrado Previsional Argentino (SIPA). Con la información de dicha fuente, se presenta la evolución de la cantidad de firmas registradas en la actividad, así como de los puestos de trabajo correspondientes en el período 2006-2016.

La desagregación de la actividad Fabricación (vinculado al segmento primario) y a la Venta y Reparación (vinculado al segmento secundario) ha permitido avanzar en un panorama general de la trama automotriz. ${ }^{2}$ La categorización del tamaño de las empresas y su respectivo nivel de puestos de trabajo registrados computa a las firmas que presentan actividad al finalizar el año y con el empleo promedio declarado. La publicación se realiza en base a las empresas que contienen empleados en relación de dependencia (es decir, que cuentan con aportes y contribuciones patronales). En lo que refiere a las empresas las mismas se catalogan en base al Código Único de Identificación Tributaria (CUIT). Las categorías de micro, pequeñas, medianas y grandes empresas son determinadas por el volumen máximo de ventas para las mismas, definido en base a criterios de productividad laboral media sectorial. ${ }^{3}$

En segundo lugar, las características sociodemográficas (categoría ocupacional y edad) y la calidad del empleo (registro en la seguridad social) en la trama automotriz en el período 2006-2016 fueron estudiadas a partir del procesamiento de la base Encuesta Permanente de Hogares del INDEC, particularmente de los 4. ${ }^{\circ}$ trimestres de los años comprendidos. Nótese que, dado que los valores de EPH son una estimación sobre la base del relevamiento en los aglomerados cubiertos por la encuesta, se vuelve necesario interpretar a los porcentajes presentados como estimaciones y no como datos absolutamente definitorios. ${ }^{4}$

En cuanto a la caracterización del perfil de la fuerza de trabajo empleada, para realizar el recorte de la trama productiva automotriz se toman en consideración los cambios introducidos en la construcción del instrumento de clasificación de ocupaciones. El INDEC utiliza el Clasificador Nacional de Ocupaciones (CNO-versión 2001). Hasta el cuarto trimestre de 2010 el INDEC construyó las categorías ocupacionales a partir de la Clasificación de actividades económicas para encuestas sociodemográficas del MERCOSUR (CAES-MERCOSUR). A partir del primer trimestre de 2011 entró en vigor la Clasificación de Actividades Económicas para Encuestas Sociodemográficas del MERCOSUR Caes - Mercosur 1.0 Versión Argentina. En 
este nomenclador se modifican ciertas denominaciones. En todos los casos la selección se refirió a la actividad automotriz, no se retoma lo vinculado a motocicletas y accesorios.

En lo que respecta a la calidad del empleo se retoma la declaración de percibir (o no) aportes jubilatorios como índice de registro en la seguridad social. En este sentido, los valores presentados corresponden a la población asalariada. La delimitación a partir de la calificación ocupacional se realiza en base a determinadas anuencias metodológicas por parte de organismos oficiales y expertos (Bertranou y Casanova, 2015).

Para complementar el análisis de la calidad del empleo se recuperan las remuneraciones promedias anuales del sector privado y los segmentos abordados. A partir del crecimiento constante de sus valores, las remuneraciones que se presentan pertenecen a los datos elaborados por el Observatorio de Empleo y Dinámica Empresarial - DGEYEL - SSPTYEL (MTEySS). Los mismos corresponden a los promedios anuales en valores corrientes en pesos. La conversión presentada es en dólares estadounidenses. ${ }^{5}$

\subsection{Las fases del periodo $2006-2016$}

La bibliografía relacionada a los ciclos económicos presenta diversas aristas. Desde diversas perspectivas a nivel regional, se ha dado una revisión a las caracterizaciones clásicas de las tendencias del mercado de trabajo vinculadas a la estructura económica, ciclo económico y composición de la fuerza de trabajo (Jiménez Barrera, 2014). En Argentina, diversos autores han definido a los ciclos económicos en relación con el papel e inserción de la producción local en el mercado mundial y las fluctuaciones que ello conlleva en el nivel de exportaciones, empleo y sus oscilaciones (Ferrer, 1995, p. 17). Con relación al vínculo entre ciclo económico y mercado de trabajo, en particular en el nuevo milenio, los cambios en la estructura ocupacional han dado lugar a estrategias de reproducción por parte de los hogares de carácter diferenciados segn las diversas fases macroeconómicas (Salvia y Vera, 2012).

De este modo y a los fines del artículo se entiende por ciclo económico a un periodo determinado de tiempo en el cual se analiza la capacidad industrial empleada y su generación de valor agregado. En este sentido dentro de un ciclo económico existen distintas fases. La fase ascendente es aquella donde una actividad presenta un relativo nivel de crecimiento constante, en el cual una fase descendente implica una caída en sus valores llegando a niveles similares al principio del ciclo económico.

Al observar las Cuentas Nacionales cabe analizar los Agregados macroeconómicos (PIB) para dar cuenta de la evolución de la trama abordada. Si se toman los datos elaborados por el INDEC la trama se nuclea dentro de dos grandes ramas de actividad: industria manufacturera (integra la rama de producción industrial donde aparece la fabricación de vehículos automotores, remolques y semirremolque como el mayor nivel de desagregación) y comercio mayorista, minorista y reparación (cuyo sector se compone de el mantenimiento y reparación de vehículos automóviles, el comercio mayorista/minorista y reparación de efectos personales y utensilios y mobiliarios domésticos). En primera instancia, la evolución de la actividad de producción de automotores es plausible de analizarse en valores anuales en millones de pesos en base al precio del año 2004.

En el gráfico 1 se observa la evolución de los valores de producción y agregado bruto. Cabe aclarar que el Valor Agregado Bruto (VAB) a precios básicos consiste en la producción tasada a precios básicos menos el gasto intermedio valorado a precio de comprados, conformándose así por a) remuneración de los asalariados; b) ingreso mixto bruto; c) excedente neto de explotación; d) consumo de capital fijo; e) otros impuestos netos de subsidios a la producción (INDEC, 2016, p. 10). La desagregación corresponde a la actividad Fabricación de vehículos automotores, remolques y semirremolques. Si bien en términos constantes la actividad ha presentado una ampliación de sus márgenes diferenciado, al analizar el valor a base 2004 se observa un decaimiento de sus términos. Fundamentalmente al finalizar el periodo los valores se mantienen casi inalterables e, incluso, el valor agregado bruto se contrae. En este contexto, según los datos disponibles del Indicador de la Utilización de la Capacidad Instalada en la Industria (el cual permite contar con un 
registro de medición proporcional de la utilización efectiva de la capacidad industrial existente), el período 2010-2016 muestra dos trayectos bien diferenciados. En los años trascurridos entre 2010-2012 la utilización de la capacidad instalada automotriz presentó niveles altos, llegando a un pico del 73,8 \% en 2011 para luego descender y estabilizarse en el $67 \%$ aproximadamente. En este sentido, la fase ascendente del ciclo presenta en los diversos indicadores un proceso de crecimiento casi constante. El proceso de contracción de la fase descendente encuentra en el 2014 una brusca caída que lleva a la industria al $51 \%$, para continuar su descenso hasta el 47,1 \% dos años después, en donde el VAB cae incluso por debajo del punto de origen.

\section{GRÁFICO 1}

Evolución del Valor Bruto de Producción (VBP), Valor Agregado

Bruto (VAB) a precios básicos y utilización de la Capacidad Instalada.

* Segmento terminal de la industria automotriz. Periodo 2006-2016



Fuente: Elaboración propia en base a datos de Cuentas Nacionales (INDEC) y en base a los datos de los informes técnicos sobre Utilización de la Capacidad Instalada en la Industria (INDEC).

Una primera caracterización de la situación laboral en el período de posconvertibilidad muestra una mejoría en las condiciones del empleo y la distribución del ingreso. Si bien los avances presentados se encuentran en el marco del movimiento de crecimiento del nivel de consumo del mercado interno, los condicionamientos estructurales del régimen de acumulación local mostraron restricciones en la capacidad de expansión, generando contracciones macroeconómicas que delimitaron las tendencias acontecidas (Lindenboim, 2015). Este escenario impactó negativamente en el mundo del trabajo, ya que la restricción en la capacidad de valorización por parte del entramado privado local -al no contar con la posibilidad de competir con los valores medios internacionales- tuvo su correlato en las condiciones del empleo (Graña, 2015), lo cual vio matizarse según su inserción sectorial (Salvia, Vera y Poy, 2015).

La industria automotriz en Argentina ha contado con diversos escenarios a lo largo de los ciclos económicos locales y globales que se sucedieron. Acompañando a la tendencia general de la estructura económica del país, la trama productiva automotriz presentó momentos de crecimiento diferenciados en sus diversos componentes, tanto en el volumen como en el registro de empleo. Schorr (2012) explica la tendencia general por un proceso de "reindustrialización acotada", lo cual genero un doble movimiento de asentamientos de la expansión de las ramas de mayor peso (entre ellas la automotriz) por un lado, y por otro el crecimiento de las pequeñas y medianas empresas (pymes). 
Con la evolución que tuvo la industria automotriz local, y su esperable correlato en su nivel de empleo, cabe preguntarse: ¿cómo evolucionó la dinámica ocupacional para los diversos segmentos laborales de la trama productiva automotriz en este contexto?

\section{Dinámica interna de la organización DEL trabajo en la trama productiva AUTOMOTRIZ}

En el desarrollo de la dinámica ocupacional se da un conjunto de desigualdades sociales que configuran un entramado de condicionantes, contextos, construcción de subjetividades e imaginarios que confluyen y entran en tensión como reflejo de múltiples factores y dimensiones. El retomar enfoques heterodoxos (aunque no necesariamente novedosos), permite ampliar la gama de herramientas conceptuales para comprender el isomorfismo de la organización social del trabajo, la dinámica de su funcionamiento y cuál es el peso de los factores tecnológicos e institucionales (Neffa, 2008). Este artículo parte de analizar la diferenciación de los puestos de trabajo, por lo que resulta necesario conceptualizar de qué manera la segmentación y dinámica ocupacional genera una dualidad en estos.

Cuestionando la visión neoclásica de un mercado de trabajo libre y único donde oferta y demanda se ajustaban tendiendo a un equilibrio, y conocidos como "dualistas", numerosos trabajos propusieron analizar los diversos puestos de trabajo existentes y de qué manera esta inserción de los puestos en la actividad económica generaba una gama de empleos de diversa calidad. Dentro de esta óptica la división se da de la siguiente manera: por un lado en un sector primario, en donde se encuentran los puestos de trabajo con mayores niveles salariales, cobertura de seguridad social, sindicalización y estabilidad laboral, siendo ocupados en grandes establecimientos y empresas de gran volumen de capital. Por el otro el sector secundario, compuesto por puestos de trabajo de baja calificación, niveles de registro y salarios disminuidos y falta o ausencia de reglamentaciones laborales, acción gremial colectiva, y ocupados en establecimientos con baja escala tecnológica. Así, la división en circuitos de puestos diferenciados marca la inexistencia de un mercado único, en donde las expectativas, comportamientos y calidades de los puestos son distintos para los actores y agentes económicos. Es decir, la estructura económica conforma una amplia gama de puestos de trabajo con diversas pautas, a lo que se lo denomina mercado de trabajo dual (Piore, 1983, p. 15). Esta división del mercado de trabajo no es coordinada, sino que los puestos de trabajo responden a lógicas desconectadas y fraccionadas.

En este esquema analítico el nivel de empleo y el salario corresponden a fenómenos esencialmente vinculados a la dinámica de la segmentación pero que se definen en procesos distintos. Esto se vincula a la pertenencia del puesto de trabajo al mercado abierto o cerrado. Esta posibilidad de empleo-desempleo afecta de manera diferente a los distintos grupos sociodemográficos a partir de las funciones económicas que desempeñan. En cuanto al salario, los procesos en que se determina exceden los elementos meramente económicos, ya que la institución salarial cumple una función social.

En base a las consideraciones del mercado de trabajo dual, en este artículo se retoman tres elementos principales para la caracterización de la segmentación laboral en la trama productiva. Se divide a los segmentos en base a los tipos de empresas (tamaño-cantidad de puestos de trabajo), las características sociodemográficas (categoría ocupacional y características sociodemográficas) y la calidad del empleo (registro en la seguridad social en base a la calificación y tamaño del establecimiento y remuneraciones).

De este modo se define un segmento primario compuesto por puestos de trabajo dedicados a la producción, encarnados por los trabajadores de terminales y empresas autopartistas empleados en grandes establecimientos, con altos registros en la seguridad social y remuneraciones por encima de la media. El segmento secundario se compone por establecimientos pymes con actividades de tipo de servicio, constituyendo una masa laboral con baja protección social y remuneraciones disminuidas en comparación con el segmento superior. En este segmento además del trabajador de los pequeños establecimientos se suma la 
figura del trabajador autónomo. En los siguientes apartados se analiza la evolución de los segmentos laborales delimitados a partir de datos declarados por las empresas en el Sistema Previsional Argentino (SIPA).

Los puestos de trabajo en términos absolutos arrojaron un crecimiento durante el período abordado (gráfico 2). Para el segmento primario la ondulación presenta su cresta en 2011 para el total de la actividad como para los diversos tramos de establecimiento. Si bien en el período descendente se observa una reducción de la plantilla, entre los extremos se da un mayor volumen en sus totales. En cuanto al segmento secundario el crecimiento se ha presentado de manera constante aunque con una intensidad distinta. Durante la fase ascendente el crecimiento de ambos segmentos arroja el periodo de menor diferencia porcentual entre sí, siendo que al cierre del ciclo es donde las diferencias en las participaciones se acrecentaron. La dinámica adquirida por el empleo durante las diversas fases del ciclo económico arroja un panorama de mayor crecimiento para el segmento secundario, tanto en términos absolutos como de diferencias porcentuales (gráfico 2). En el período 2006-2016 el total del stock automotor circulante ha crecido de manera sostenida, duplicando la cantidad de unidades producidas a finales de la década (14.134.565). Compuesto por una amplia gama de tipos de automotores, el peso preponderante de los automóviles pequeños ha sido en términos totales y relativos considerable: prácticamente el $80 \%$ del total del stock circulante durante todo el período corresponde a este tipo (ver anexo cuadro 1).

\section{GRÁFICO 2}

Evolución de los puestos de trabajo para el segmento primario y secundario para la trama productiva automotriz. Valores absolutos, porcentajes y diferencias porcentuales. Periodo 2006-2016.

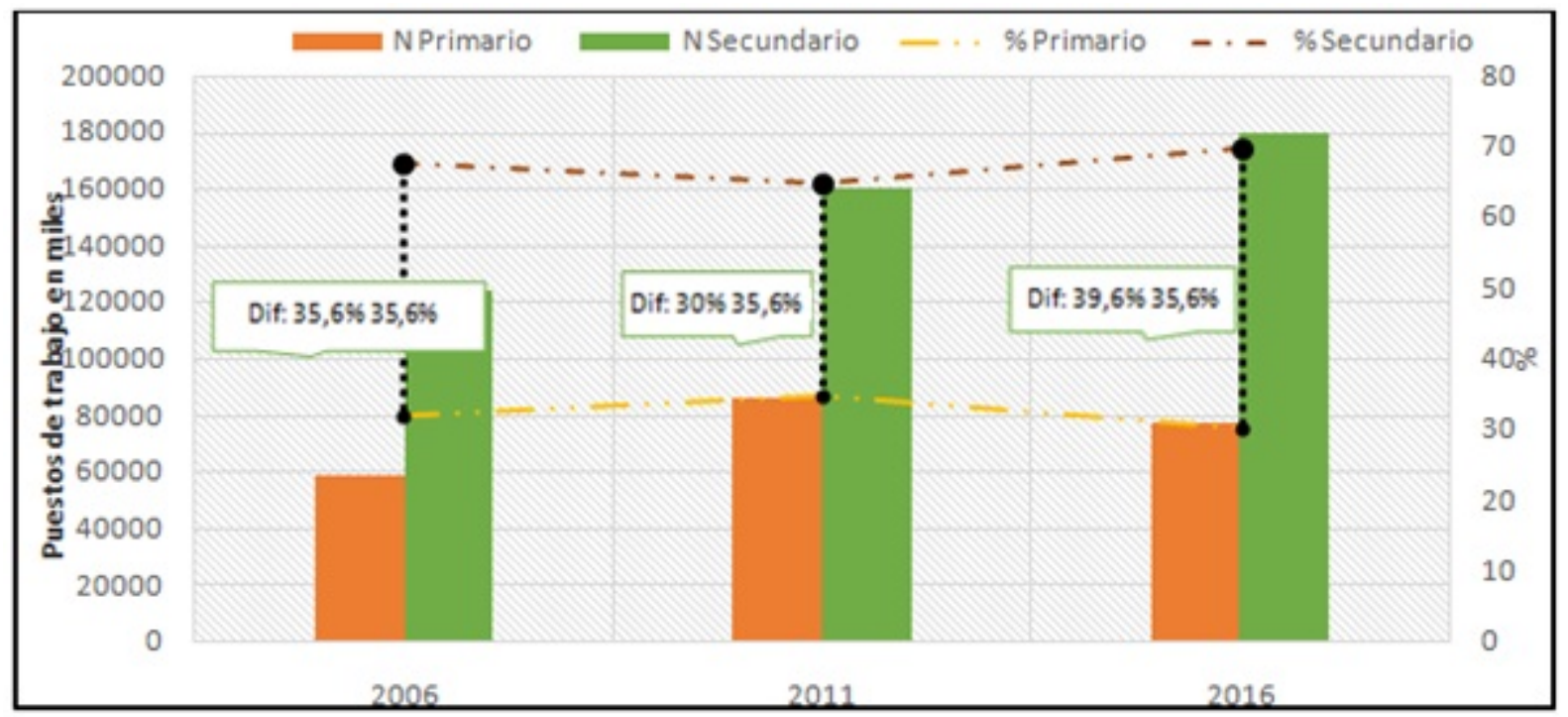

Fuente: Elaboración propia en base a datos de OEDE-DGEYEL - SSPTYEL.

Con un panorama diferenciado entre los segmentos en los siguientes apartados se presenta el desarrollo interno en cuanto a la evolución de los tipos de las empresas, las características sociodemográficas y la calidad del empleo.

\subsection{Segmento primario}

Para analizar las características de la demanda de trabajo en el cuadro 1 se encuentran los datos de la cantidad de empresas y puestos de trabajos. Para el total del segmento primario dentro del ciclo de expansión el 2011 fue el momento de mayor cantidad de empresas, situación que presenta un lento retroceso hasta el 2016 donde registra 1.538 firmas. El proceder de las empresas micro, pequeñas y medianas muestra una ondulación que acaba con valores un tanto menores en sus extremos. Las microempresas ven 
declinar casi de manera constante su cantidad. En la actividad del segmento primario se observa que en las empresas más grandes el volumen de puestos de trabajo logró un incremento de sus plantillas. En el resto, si bien la caída no es sumamente fuerte, sí se observa un primer momento de incremento para comenzar un escalonado descenso.

Los puestos de trabajo en términos absolutos arrojaron un crecimiento. La ondulación presenta su cresta en 2011 tanto para el total de la actividad como para los diversos tramos de establecimiento. La concentración de trabajo en las mayores empresas muestra el peso preponderante de las grandes firmas terminales. La capacidad instalada de las mismas refleja la expansión de la actividad en los puestos de trabajo, así como su contracción a finales del período. La caracterización del segmento con grandes establecimientos con un volumen de capital y tecnología preponderante se vuelve visible en los datos presentados.

\section{CUADRO 1}

Evolución de la inserción del puesto de trabajo en la trama productiva automotriz para el segmento primario. Empresas activas al cierre de año. Desagregado a 2 dígitos del CIIU. Total país 2006-2016.

\begin{tabular}{|c|c|c|c|c|c|c|}
\hline \multirow{3}{*}{$\begin{array}{c}\text { Tamaño del } \\
\text { establecimiento }\end{array}$} & \multicolumn{6}{|c|}{ Año } \\
\hline & \multicolumn{2}{|c|}{2006} & \multicolumn{2}{|c|}{2011} & \multicolumn{2}{|c|}{2016} \\
\hline & $\begin{array}{l}\text { Cantidad } \\
\text { de } \\
\text { empresas }\end{array}$ & $\begin{array}{l}\text { Puestos } \\
\text { de trabajo }\end{array}$ & $\begin{array}{l}\text { Cantidad } \\
\text { de } \\
\text { empresas }\end{array}$ & $\begin{array}{l}\text { Puestos } \\
\text { de } \\
\text { trabajo }\end{array}$ & $\begin{array}{l}\text { Cantidad } \\
\text { de } \\
\text { empresas }\end{array}$ & $\begin{array}{l}\text { Puestos } \\
\text { de } \\
\text { trabajo }\end{array}$ \\
\hline Micro & 805 & 2.699 & 788 & 2.549 & 737 & 2.361 \\
\hline Pequeñas & 523 & 7.660 & 530 & 7.663 & 474 & 6.914 \\
\hline Medianas & 199 & 9.740 & 210 & 10.758 & 195 & 9.231 \\
\hline Grandes & 126 & 39.041 & 146 & 65.379 & 132 & 59.396 \\
\hline Total & 1.653 & 59.139 & 1.674 & 86.348 & 1.538 & 77.901 \\
\hline
\end{tabular}

Fuente: Elaboración propia en base a datos de OEDE-DGEYEL - SSPTYEL.

Cabe resaltar que la composición de las firmas encuentra una dinámica particular. El asentamiento de terminales automotrices es consecuencia de estrategias globales de las casas matrices. En el marco de la contracción de la industria automotriz, en el segmento primario la situación se presenta dispar al interior de las diversas empresas que podrían comprender la actividad. Al observar la trama automotriz y disgregar los datos de la evolución de la trama en tres dígitos del CIUU se hacen presentes las actividades que componen la gama de actividades locales de producción. Sin grandes cambios en sus valores la fabricación de automotores ven reducir su número. Es en la fabricación de autopartes en donde la caída resulta más sensible, con una reducción del $9 \%$ (ver anexo cuadro 2 ). 
CUADRO 2

Características sociodemográficas del segmento primario. Distribución porcentual. Total de los aglomerados urbanos. Período 2006-2011-2016**

\begin{tabular}{|c|c|c|c|c|c|}
\hline \multirow{2}{*}{\multicolumn{3}{|c|}{ Segmento primario }} & \multicolumn{3}{|c|}{ Año } \\
\hline & & & 2006 & 2011 & 2016 \\
\hline \multirow{11}{*}{$\begin{array}{l}\text { Características } \\
\text { demográficas }\end{array}$} & \multirow{4}{*}{ Categoría } & Patrón & $2 \%$ & $0,2 \%$ & $0 \%$ \\
\hline & & Cuenta propia & $4 \%$ & $3 \%$ & $1,6 \%$ \\
\hline & & Obrero o empleado & $94 \%$ & $96 \%$ & $98,4 \%$ \\
\hline & & $\begin{array}{l}\text { Trabajador familiar sin } \\
\text { remuneración }\end{array}$ & - & - & - \\
\hline & \multirow{4}{*}{$\begin{array}{l}\text { Grupos } \\
\text { etarios }\end{array}$} & 18 a 29 & $40 \%$ & $32 \%$ & $18 \%$ \\
\hline & & 30 a 45 & $34 \%$ & $44 \%$ & $43 \%$ \\
\hline & & 46 a 59 & $23 \%$ & $17 \%$ & $33 \%$ \\
\hline & & 60 y más & $4 \%$ & $7 \%$ & $6 \%$ \\
\hline & \multirow{3}{*}{ Calificaciones } & Técnica & $6 \%$ & $11 \%$ & $24 \%$ \\
\hline & & Operaria & $84 \%$ & $71 \%$ & $71 \%$ \\
\hline & & Sin calificación & $9 \%$ & $5 \%$ & $1 \%$ \\
\hline \multirow{4}{*}{$\begin{array}{l}\text { Registro en la } \\
\text { seguridad } \\
\text { social }\end{array}$} & \multirow{3}{*}{ Calificaciones } & Técnica & $100 \%$ & $87,3 \%$ & $100 \%$ \\
\hline & & Operaria & $85,4 \%$ & $93,8 \%$ & $95,1 \%$ \\
\hline & & Sin calificación & $33,1 \%$ & $100 \%$ & $0 \%$ \\
\hline & \multicolumn{2}{|c|}{ Total ocupados con registro } & $81,3 \%$ & $94,1 \%$ & $96,5 \%$ \\
\hline
\end{tabular}

Fuente: Elaboración propia en base a datos de la EPH-INDEC, 4 trimestre.

Al observar la composición sociodemográfica en trama automotriz vinculada al segmento primario en el período 2006-2016 (cuadro 2), en términos absolutos se presenta una hegemonía preponderante de obreros a lo largo de todo el período. Retomando los valores de la categoría ocupado, esta no solo se presenta en términos generales como la categoría predominante, ya que en promedio representa el $90 \%$ de la población a lo largo de la década, sino que además esta categoría presenta porcentajes más elevados a medida que la masa total se reduce.

La composición de los distintos grupos etarios a partir de las funciones económicas que desempeñan encuentra en los segmentos abordados un ejemplo de ello. En el segmento primario el rango etario con mayor peso es el perteneciente a la franja etaria de 30 a 45 años, pasando del $34 \%$ en 2006, $44 \%$ en 2011, para luego retroceder al $43 \%$ en 2016. Las personas de 46 a 59 años comienzan en 2006 con un $23 \%$, finalizando con un fuerte incremento en 2016 (33\%). De este modo puede observarse cómo el segmento encuentra su principal grupo etario en la franja de 30 a 45 años. Siendo principalmente operario, el incremento de la calificación técnica la posiciona como la segunda categoría en tendencia de crecimiento. 
Los altos niveles de registro de aportes jubilatorios, para el total de la población del segmento, presentan valores que no descienden prácticamente del $88 \%$ y que alcanzan registros del $100 \%$ en algunos períodos. Las categorías operaria y técnicas presentan valores iguales o por encima del registro de aportes del total de la población. La tendencia general se asocia a la población con calificación operaria, en donde sus valores presentan una estimación cercana.

Al introducirse el tramo de empleo en la remuneración para los segmentos a lo largo de la década seleccionada, las remuneraciones promedio del segmento primario perteneciente a las empresas de más de 200 empleados se han mantenido por encima de su par, como también del promedio total del sector privado. Las brechas entre las categorías y el total del sector privado con los salarios de las mayores empresas automotrices son algo constante en todo el período. El promedio salarial en las empresas de más de 200 trabajadores es superior al total del sector privado, e incluso llega superar esa brecha a la categoría con respecto a las remuneraciones promedio de las empresas de hasta 9 trabajadores. Al efectuar el mismo análisis, pero esta vez focalizado únicamente en las empresas del segmento primario, se observa cómo nuevamente las tendencias anteriores se replican, generando brechas salariales considerables entres las grandes empresas y los demás segmentos, incluso los valores promedios totales (ver anexo cuadro 3 ).

\subsection{Segmento secundario}

El segmento secundario presenta un crecimiento para luego entrar en una meseta de alrededor de 21.600 empresas siendo que la brecha se amplía en favor de las pequeas y microempresas. El ciclo expansión/ contracción arrojó un crecimiento en términos absolutos como relativos. Si bien esta situación es lo que se repite en las cuatro categorías, las empresas más grandes muestran un nivel de crecimiento considerable. Las otras categorías presentan un comportamiento similar. No obstante, en las microempresas es donde se refleja de manera absoluta la tendencia total, ya que en este segmento es donde se concentra aproximadamente el $80 \%$ de la totalidad de las empresas.

Al observar el total de los puestos de trabajo del segmento, con más de 55.000 nuevos puestos de trabajo para el total de la actividad en 2016 con respecto a 2006, al interior de cada una de las categorías de empresas las intensidades del incremento fueron diversas. Aunque la regla general fue hacia el alza, el ascenso no ha sido de manera pareja, mostrando dos tipos de incrementos. El ciclo de contracción, si bien los incrementos de las empresas son bastante más moderados, cabe resaltar que la cantidad de puestos aumenta en similares proporciones. Es así como el comportamiento generalizado ha sido hacia un incremento en la cantidad de los puestos de trabajo. A diferencia del segmento primario, en este segmento la distribución heterogénea de la cantidad de ocupados registrados presenta un escenario distinto.

CUADRO 3

Evolución de la inserción del puesto de trabajo en la trama productiva automotriz para el segmento secundario. Empresas activas al cierre de año. Desagregado a 2 dígitos del CIIU. Total país 2006-2016.

\begin{tabular}{|l|c|c|c|c|c|c|}
\hline \multirow{2}{*}{$\begin{array}{l}\text { Tamaño del } \\
\text { establecimiento }\end{array}$} & \multicolumn{5}{|c|}{2006} & \multicolumn{2}{c|}{ Año } \\
\cline { 2 - 8 } & $\begin{array}{l}\text { Cantidad de } \\
\text { empresas }\end{array}$ & $\begin{array}{l}\text { Puestos de } \\
\text { trabajo }\end{array}$ & $\begin{array}{l}\text { Cantidad } \\
\text { de } \\
\text { empresas }\end{array}$ & $\begin{array}{l}\text { Puestos } \\
\text { de } \\
\text { trabajo }\end{array}$ & $\begin{array}{l}\text { Cantidad } \\
\text { de } \\
\text { empresas }\end{array}$ & $\begin{array}{l}\text { Puestos } \\
\text { de } \\
\text { trabajo }\end{array}$ \\
\hline Micro & 12.855 & 22.564 & 14.183 & 25.246 & 14.116 & 25.441 \\
\hline Pequeñas & 5.359 & 54.887 & 6.066 & 62.486 & 6.177 & 64.842 \\
\hline Medianas & 653 & 25.182 & 834 & 32.288 & 919 & 35.700 \\
\hline Grandes & 201 & 21.799 & 337 & 40.411 & 417 & 53.748 \\
\hline Total & 19.068 & 124.432 & 21.420 & 160.429 & 21.629 & 179.730 \\
\hline
\end{tabular}

Fuente: Elaboración propia en base a datos de OEDE-DGEYEL - SSPTYEL. 
Queda en evidencia cómo el peso preponderante se encuentra en las actividades vinculadas a los servicios, siendo la producción un número bastante menor en comparación. Mención aparte merece el grupo de empresas dedicadas al mantenimiento y reparación de vehículos, ya que a lo largo de la década representa cambios que esencialmente la convierte en la actividad más convocante. La evolución de la demanda de trabajo presentó que las actividades que mayor crecimiento en su cantidad de empresas fueron Mantenimiento y reparación de vehículos automotores, excepto motocicletas y Venta al por menor de partes, piezas y accesorios de vehículos automotores. Con un ritmo de crecimiento menor se presentan las actividades de Venta al por menor de combustible para vehículos automotores y motocicletas y Venta de vehículos automotores nuevos, excepto motocicletas y Venta al por mayor de partes, piezas y accesorios de vehículos automotores. Más bien se ha centralizado en la actividad de Mantenimiento de vehículos y Venta de autopartes en detrimento de aquellos que se nuclean en la actividad de Fabricación (cuadro 3).

\section{CUADRO 4}

Características sociodemográficas del segmento secundario. Distribución porcentual. Total de los aglomerados urbanos. Período 2006-2011-2016.

\begin{tabular}{|c|c|c|c|c|c|}
\hline \multicolumn{3}{|c|}{ Segmento secundario } & \multicolumn{3}{|c|}{ Año } \\
\hline & & & 2006 & 2011 & 2016 \\
\hline \multirow{11}{*}{$\begin{array}{l}\text { Características } \\
\text { demográficas }\end{array}$} & \multirow{4}{*}{ Categoría } & Patrón & $10 \%$ & $9 \%$ & $12 \%$ \\
\hline & & Cuenta propia & $34 \%$ & $41 \%$ & $44 \%$ \\
\hline & & Obrero o empleado & $54 \%$ & $49 \%$ & $43 \%$ \\
\hline & & $\begin{array}{l}\text { Trabajador familiar sin } \\
\text { remuneración }\end{array}$ & $2 \%$ & $1 \%$ & $0,4 \%$ \\
\hline & \multirow{4}{*}{$\begin{array}{l}\text { Grupos } \\
\text { etarios }\end{array}$} & 18 a 29 & $26 \%$ & $43 \%$ & $36 \%$ \\
\hline & & 30 a 45 & $15 \%$ & $40 \%$ & $37 \%$ \\
\hline & & 46 a 59 & $39 \%$ & $14 \%$ & $17 \%$ \\
\hline & & 60 y más & $20 \%$ & $2 \%$ & $10 \%$ \\
\hline & \multirow{3}{*}{ Calificaciones } & Técnica & $3 \%$ & $2 \%$ & $1 \%$ \\
\hline & & Operaria & $72 \%$ & $75 \%$ & $70 \%$ \\
\hline & & Sin calificación & $25 \%$ & $30 \%$ & $28 \%$ \\
\hline \multirow{4}{*}{$\begin{array}{l}\text { Registro en la } \\
\text { seguridad } \\
\text { social }\end{array}$} & \multirow{3}{*}{ Calificaciones } & Técnica & $75,9 \%$ & $22,1 \%$ & $40,6 \%$ \\
\hline & & Operaria & $43 \%$ & $55,5 \%$ & $48,4 \%$ \\
\hline & & Sin calificación & $13,1 \%$ & $15,7 \%$ & $9 \%$ \\
\hline & \multicolumn{2}{|c|}{ Total ocupados con registro } & $37,3 \%$ & $45,6 \%$ & $37,3 \%$ \\
\hline
\end{tabular}

Fuente: Elaboración propia en base a datos de la EPH-INDEC, 4 trimestre.

La trama productiva automotriz ha presentado en Argentina a lo largo del período 2006-2016 un crecimiento acotado. La creación de puestos de trabajo en la trama centrada en las actividades de servicios y mantenimientos se asoma como un elemento destacado, ya que el crecimiento de las actividades de mantenimiento y reparación de vehículos y la venta de autopartes muestra un desenvolvimiento de micro establecimientos que concentraron un porcentaje significativo de la masa laboral. Es decir, el sector servicios crece en comparación al sector de producción, tendencia que se ve acompañada con el contexto económico posconvertibilidad (ver anexo cuadro 2). La dinámica en las características sociodemográficas presentó dentro del segmento secundario, tanto en la categoría ocupacional como para los grupos etarios, una diferenciación sustancial con el segmento primario.

Al observar las características sociodemográficas resalta que entre la categoría Cuenta propia y Ocupado a lo largo del período es que se encuentran más del $85 \%$ de la población (cuadro 4). Al principio de la década la superioridad porcentual de los obreros ha estado por encima del $50 \%$; esta superioridad comienza con un 
retroceso proporcional a la presencia del cuentapropismo. Este se vuelve significativo, llegando al $41 \%$ en el 2011 y superando a la cantidad de obreros en el 2016, obteniendo el cuentapropismo a un total del $44 \%$ y $43 \%$ respectivamente. En este sentido, el ciclo de expansión/contracción sostuvo un proceso de incremento de figuras laborales con baja protección social.

La población ocupada en el segmento, en tanto, presenta en los grupos de edad una composición más heterogénea. De igual modo que en el segmento primario, este encuentra en el grupo de 30 a 45 el segmento de mayor participación, ya que pasa del $23 \%$ en 2006 (momento de menor participación) para empezar un crecimiento sostenido hasta obtener el $40 \%$ en 2011 y luego quedar en $37 \%$ en 2016. No obstante, si se toma al conjunto de trabajadores de 18 a 29 años estos presentan una mayor participación, lo que lo constituye en el grupo etario de mayor presencia, quedando casi empatado en la distribución porcentual. Aunque la proporción de operarios se encuentra en sintonía con el segmento primario, la fuerza de trabajo sin calificación se presenta como el segundo grupo con estabilidad a lo largo del período.

El segmento presenta valores de registro en la seguridad social sustancialmente menores para el total de las actividades. Todas las categorías presentan una considerable reducción frente a sus pares del otro segmento. Cabe destacar que aquellos ocupados que no presentan calificación arrojan niveles de registro muy bajos, y que incluso en la tendencia de la década se vieron aún más perjudicados.

Si bien el comportamiento de los segmentos de empresas ha tenido una regularidad de mantención y profundización de las diferencias intra-tramos, solo en 2016 las brechas entre las remuneraciones de quienes se emplean en el segmento secundario en establecimiento de hasta 9 empleados ha visto trastocar la lógica expuesta. En la misma línea de análisis, solo que esta vez circunscripto al segmento secundario, las tendencias al crecimiento se mantienen al igual que en el caso anterior. No obstante, en esta ocasión, las remuneraciones promedias para las grandes empresas son superiores a los demás segmentos y al promedio de la rama. En cuanto a la brecha entre las empresas de mayor número y las más pequeñas, la misma es considerable, y es a partir de 2011 cuando comienza a despegarse para mantener valores similares en los próximos años. Al tener en cuenta los promedios de los segmentos y el total del sector primado, la trama productiva automotriz presenta una leve supremacía en sus niveles remunerativos. El segmento secundario cuenta en su promedio, en lo que respecta a las remuneraciones, un posicionamiento más favorable (ver anexo cuadro 3).

\section{Reflexiones finales. Trama productiva automotriz: ciclo económico y SEGMENTACIÓN LABORAL}

La trama productiva automotriz ha presentado a lo largo del período 2006-2016 un crecimiento que arrojó diversas fases. Enfocando en el total de la industria, se comprueba un incremento en la cantidad de sus puestos de trabajo, con características diferenciales al interior de los segmentos que la componen. En esta dirección, se pudo observar un proceso de concentración para el segmento primario, que se evidencia en que si bien el empleo crece en un $24 \%$, su capacidad instalada se reduce; es decir, se observa un incremento en cantidad de puestos en menor cantidad de empresas de gran volumen. Con una expansión del $30 \%$ de la plantilla, el crecimiento del segmento secundario se da tanto en términos absolutos como relativos en lo que refiere a la cantidad de empresas y puestos de trabajo. Si bien la plantilla presentó una expansión mucho más acentuada en término absolutos, en la fase descendente este segmento no tiende a la concentración sino más bien a una distribución heterogénea. A diferencia de lo que ocurre con el segmento primario, el segmento secundario en la fase descendente del ciclo presenta un crecimiento acotado (pero crecimiento al fin) en sus puestos de trabajo y empresas involucradas. Esta situación tiende a matizar con mayor fuerza a partir de las características propias de la actividad.

En cuanto a la composición sociodemográfica puede referirse que la trama productiva automotriz presenta una población en plena edad económica activa. Si bien en el segmento primario son los trabajadores adultos (30 a 45 años y 46 a 59) quienes predominan, en la actividad vinculada al segmento secundario son los 
trabajadores jóvenes (hasta 29 años) quienes se posicionan junto a los adultos de hasta 45 años como los grupos etarios fundamentales, sobre el final del período. Haciendo evidente que, frente al ciclo recesivo y la contracción de las empresas grandes, las personas jóvenes encontraron ocupaciones en el segmento secundario.

La calidad del empleo presentó una diferenciación entre los segmentos. Esta diferencia en los niveles de protección social es una característica de un mercado segmentado. A su vez, el peso del cuentapropismo en el segmento secundario abona a una heterogeneidad mayor en las figuras laborales características de la oferta de trabajo. Dentro de los asalariados las diferencias en los registros de la seguridad social son notorias, tanto en las diversas calificaciones como en el tamaño del establecimiento de inserción del ocupado. Al observar el promedio de remuneraciones para ambos, las empresas abordadas y el total del sector privado, se presenta una clara brecha entre quienes se emplean en los segmentos, siendo el primario muy superior a partir de la concentración de los puestos de trabajo. Si bien las diferencias no son tan marcadas, al tomar el promedio del sector privado y el total del segmento secundario se observa cómo en esta última los valores son un tanto mayores a lo largo de la década. La tendencia a remuneraciones más altas por parte de las empresas de más de 200 puestos de trabajo es algo que se sostiene a lo largo de todo el período 2006-2016.

Frente a las distintas fases del ciclo y en virtud del comportamiento diferenciado de los segmentos, la idea de un mercado de trabajo único se vuelve un relato sin correlato empírico. En contraposición, diversas investigaciones dan cuenta de la necesidad de comprender la realidad laboral latinoamericana a partir de la contextualización y apreciación en grados de sus diversos fenómenos vinculados a la informalidad y precariedad, como es el caso del cuentapropismo (Busso, 2010). Cabe destacar que, para el segmento secundario, fundamentalmente es en las actividades de mantenimiento y reparación de vehículos en donde se insertan en mayor medida los puestos de trabajo, y en las cuales resulta más asimilable la idea de los "trabajos independientes".

A lo largo del artículo se presentó la evidencia de la segmentación laboral y las características de los diversos segmentos. En términos absolutos, el crecimiento que arrojó el segmento secundario y las condiciones laborales propias vuelven pertinente su observación como temática. No obstante, y a partir de dichas atenciones, surgen interrogantes. Resulta adecuado referir que en la trama productiva automotriz existe un desarrollo diferenciado entre los segmentos laborales. Sin embargo, los volúmenes de empleo son un factor que observar. Así, para el caso del segmento secundario se observa un incremento en los puestos de trabajo y una avanzada de la población más joven. Esto permite matizar la cadena de desplazamiento propio del segmento primario al secundario fruto de un proceso de contracción. En este sentido, ¿qué mecanismos influyeron en la expansión de este segmento a partir de la fase descendente del ciclo? ¿Cómo fueron los procesos de inserción ocupacional?

Al tener en cuenta el punto anterior resulta necesario considerar de qué manera el desarrollo del segmento primario influye en toda la trama productiva, volviendo ineludible analizar cómo la presión directa e indirecta ejercida impacta en el desenvolvimiento de los distintos segmentos. Continuando con esta revisión, la pertinencia que adquiere el segmento secundario se consolida en torno a su propio crecimiento, al vínculo entre los segmentos y la capacidad de presión del primero sobre el segundo en cuanto a su demanda de trabajo y a las figuras laborales que surgen. A su vez, y con relación a las figuras laborales existentes, queda abierto el interrogante: ¿con qué herramientas y capacidades cuentan los actores intervinientes para impulsar acciones dentro de esta dinámica construida?

Las diversas reflexiones e interrogantes abiertos consignan un campo de investigación a desarrollarse por parte de quienes subscriben. En este sentido, aportar al campo de los estudios del trabajo se entrelaza con el deseo de contribuir con herramientas para los actores intervinientes. Siendo la consolidación del colectivo de trabajo una de las pericias para recuperar la centralidad del trabajo como estrategia de mejora de las condiciones de vida, la misma se apunta en la necesidad de un modelo de desarrollo con justicia distributiva. 


\section{ReFERENCIAS}

Asociación de Fábricas de Automóviles (ADEFA). Anuarios. En línea http://www.adefa.org.ar/es/estadisticas-anua rios.

Bertranou, F. y Casanova, L. (coord.) (2015). Caminos hacia la formalización laboral en Argentina. Buenos Aires: Oficina de País de la OIT para Argentina.

Busso, M. (2010). Las crisis y el trabajo informal en la Argentina (o de cómo las crisis socio-económicas permean lugares de trabajo "atípicos". Revista Atlántida, 2, 125-138.

Carbajal Suárez, Y. (2010). Sector automotriz: reestructuración tecnológica y reconfiguración del mercado mundial. Paradigma Económico, [S.l.], 2(1), 24-52.

Castillo, J. (dir.) (2005). El trabajo recobrado. Una evaluación del trabajo realmente existente en España, Colección Sociología del Trabajo, Madrid: Miño y Dávila editores.

Cimoli, M. y Porcile, G. (2011). Global Growth and International Cooperation: A Structuralist Perspective. Cambridge Journal of Economics, 35, 383-400.

De la Garza, E. y Hernández Romo, M. (2018). Configuraciones productivas y laborales en la tercera generación de la industria automotriz terminal en México, México: Universidad Autónoma Metropolitana, Unidad Iztapalapa.

Ferrer, A. (1995). Los ciclos económicos en la Argentina: del modelo primario exportador al sistema de hegemonía financiera. América Latina hoy, 11, 25-30.

Fröbel, F., Heinrichs, J. y Kreye, O. (1978). The new international division of labour. Information (International Social Science Council), 17(1), 123-142.

Graña, J. (2015). Los problemas productivos de las empresas y su vinculación con el deterioro de las condiciones de empleo de los trabajadores, cap. 1 en Salvia, A. y Lindenboim, J. (2015): Hora de Balance: Proceso de Acumulación, mercado de trabajo y bienestar. Argentina 2002-2014. Buenos Aires: EUDEBA.

Guevara, S. (2011). El MERCOSUR a la luz de la industria automotriz: integración productiva, intensificación del trabajo y acumulación de capital, Studia Politicae, 23, 51-74. Recuperado de https://ri.conicet.gov.ar/bitstream/handle/11336/22397/CONICET_Digital_Nro.2df28f91-cdb4-492792c8-64b1b5bd46a0_A.pdf?sequence $=2$ \&isAllowed $=\mathrm{y}$.

Harari, I. (2015). A media máquina: procesos de trabajo, lucha de clases y competitividad en la industria automotriz argentina (1952-1976). Buenos Aires: Ediciones Razón y Revolución.

Ianni, V. (2008). La especificidad del desarrollo de la industria automotriz en la Argentina, 1959-1963. Estudos IberoAmericanos, PUCRS, 34(2), 97-113.

Instituto Nacional de Estadística y Censos - I.N.D.E.C. (2016). Cuentas nacionales: metodología de estimación: base 2004 y serie a precios constantes y corrientes. - 1a ed. - Ciudad Autónoma de Buenos Aires: Instituto Nacional de Estadística y Censos - INDEC, 2016. [En línea] Recuperado de https://www.indec.gob.ar/indec/web/Inst itucional-Indec-Publicaciones

Instituto Nacional de Estadística y Censos - I.N.D.E.C. (varios). Informes técnicos. Utilización de la capacidad instalada en la industria. Ciudad Autónoma de Buenos Aires: Instituto Nacional de Estadística y Censos - INDEC, 2016. [En línea] Recuperado de https://www.indec.gob.ar/indec/web/Institucional-Indec-InformesTecnicos-15

Jiménez Barrera, Y. (2014). Los ciclos económicos largos y su dialéctica con el desenvolvimiento capitalista. Economía y Desarrollo, 151(1), 44-55.

Lindenboim, J. (2015). Trampas del crecimiento argentino en las últimas décadas. Buscando claves interpretativas, Introducción, en Salvia, A. y Lindenboim, J. (2015): Hora de Balance: Proceso de Acumulación, mercado de trabajo $y$ bienestar. Argentina 2002-2014. Buenos Aires: EUDEBA.

Neffa, J. (dir.) (2008). Teorías económicas sobre el Mercado de Trabajo [Tomo III. Análisis institucionalistas]. Buenos Aires: Fondo de Cultura Económica.

Picabea, J. y Thomas, H. (2015). Autonomía tecnológica y desarrollo nacional: historia del diseño y producción del Rastrojero y la moto Puma. Buenos Aires: Atuel. 
Pinazo, G. (2015). El desarrollismo argentino: una mirada crítica desde la industria automotriz. Los Polvorines: Ediciones UNGS, Universidad Nacional de General Sarmiento.

Pinazo, G., Dinerstein, N. y Córdoba, F. (2017). Un aporte a la discusión sobre la productividad laboral en la Industria Argentina. Cuadernos de economía critica, 3(6), 101-125.

Piore, M. (edit.) (1983). Paro e inflación: perspectivas institucionales y estructurales. Madrid: Alianza Editorial.

Salvia, A. y Vera, J. (2012). Cambios en la estructura ocupacional y en el mercado de trabajo durante fases de distintas reglas macroeconómicas (1992-2010). Estudios del Trabajo, 41, 21-51.

Salvia, J., Vera, J. y Poy, S. (2015). Cambios y continuidades en la estructura ocupacional urbana argentina, cap. IV, en Salvia, A. y Lindenboim, J. (2015): Hora de Balance: Proceso de Acumulación, mercado de trabajo y bienestar. Argentina 2002-2014. Buenos Aires: EUDEBA.

Santarcángelo, J. y Pinazo, G. (2009). La reindustrialización en la posconvertibilidad: una mirada desde el sector automotriz. Realidad Económica, 247, 38-61

Schorr, M. (2012). Industria y neodesarrollismo en la posconvertibilidad. Voces en el Fénix, 16.

Sousa Santos, B. (2011). Epistemología del Sur. Utopia y Praxis Latinoamericana, 16(54), 17-39.

Treacy, M. (2018). Estrategias de acumulación de las cámaras empresariales automotrices argentinas y la integración productiva regional 2002-2015. Realidad económica, 47(319), 109-147.

Wlosko, M., Ros, C., Panigo, D., Rivero, P., Letcher, H., Strada, J. Neffa, J. (2016). Despidos masivos en argentina: caracterización de la situación y análisis del impacto sobre la. [En línea] Recuperado de http://www.iade.org.ar/system/files/despidos_masivos_en_argentina__caracterizacion_de_la_situacion_y_analisis_del_impacto_sobre_la_salud_fisica_y_mental_2015-2016_vfinal.pdf.

Yoguel, G. (2007). Tramas productivas y generación de ventajas competitivas: un abordaje metodológico para pasar de la firma individual a la red. En M. Novick y H. Palomino, Estructura productiva y empleo. Un enfoque transversal. Buenos Aires: MTEySS Miño y Dávila.

\section{Anexo estadístico}

\section{CUADRO 1}

Composición del stock automotor circulante según el tipo de vehículo.

Total país. Período 2006, 2011 y 2016. Valor en miles de unidades

\begin{tabular}{|c|c|c|c|c|}
\hline Segmento & Empresas & 2006 & 2011 & 2016 \\
\hline \multirow[t]{3}{*}{ Primario } & Fabricación de vehículos automotores & 19 & 21 & 17 \\
\hline & $\begin{array}{l}\text { Fabricación de carrocerías para vehículos } \\
\text { automotores; fabricación de remolques y } \\
\text { semirremolques }\end{array}$ & 302 & 313 & 311 \\
\hline & $\begin{array}{l}\text { Fabricación de partes; piezas y acces orios para } \\
\text { vehículos automotores y sus motores }\end{array}$ & 1.330 & 1.334 & 1.210 \\
\hline \multirow[t]{4}{*}{ Secundario } & $\begin{array}{l}\text { Mantenimiento y reparación de vehícul os automotores, } \\
\text { excepto motocicletas }\end{array}$ & 8.039 & 9.200 & 8.813 \\
\hline & $\begin{array}{l}\text { Venta de partes, piezas y accesorios de vehículos } \\
\text { automotores }\end{array}$ & 5.765 & 6.610 & 7.004 \\
\hline & $\begin{array}{l}\text { Venta al por menor de combustible para vehículos } \\
\text { automotores y motocicletas }\end{array}$ & 3.793 & 3.798 & 3.921 \\
\hline & Venta de vehículos automotores, excepto motocicletas & 1.519 & 1.825 & 1.891 \\
\hline
\end{tabular}




\section{CUADRO 2}

Evolución de empresas para segmento primario y secundario.

Desagregado a 3 dígitos del CIIU. Total país. Período 2006, 2011 y 2016.

\begin{tabular}{|l|c|c|c|}
\hline Tipo de vehículo & \multicolumn{3}{|c|}{ Stock circulante } \\
\cline { 2 - 4 } & 2006 & 2011 & 2016 \\
\hline Ómnibus & 62.785 & 75.891 & 85.023 \\
Vehículo de carga & 417.042 & 573.481 & 680.284 \\
Utilitario liviano & 1.370 .312 & 2.040 .269$. & 2.657 .562 \\
\hline Automóviles & 5.325 .231 & 8.269 .443 & 10.711 .696 \\
\hline
\end{tabular}

Fuente: Elaboración propia en base a datos del OEDE-DGEYEL - SSPTYEL

\section{CUADRO 3}

Remuneración promedio total de ocupados para el segmento primario, segmento secundario y total del sector privado. Empresas de más de 200 empleados y empresas de hasta 9 empleados. Total país 2006-2016.

\begin{tabular}{|c|c|c|c|c|c|c|}
\hline \multirow{3}{*}{$\begin{array}{l}\text { Tamaño de } \\
\text { empresas }\end{array}$} & \multicolumn{6}{|c|}{ Remuneración promedio } \\
\hline & \multicolumn{2}{|c|}{2006} & \multicolumn{2}{|c|}{2011} & \multicolumn{2}{|c|}{2016} \\
\hline & Primario & Secundario & Primario & Secundario & Primario & Secundario \\
\hline Más de 200 & 1200 & 726 & 2479 & 1494 & 2523 & 1533 \\
\hline $\begin{array}{l}\% \text { privado más de } \\
200\end{array}$ & \multicolumn{2}{|c|}{782} & \multicolumn{2}{|c|}{1745} & \multicolumn{2}{|c|}{1783} \\
\hline Hasta 9 & 440 & 392 & 925 & 865 & 881 & 899 \\
\hline$\%$ privado hasta 9 & \multicolumn{2}{|c|}{359} & \multicolumn{2}{|r|}{792} & \multicolumn{2}{|r|}{808} \\
\hline
\end{tabular}

Fuente: Elaboración propia en base a datos del OEDE -DGEYEL - SSPTYEL.

\section{Notas}

1 Se agradecen las observaciones y comentarios realizados en los dictámenes. Dichos aportes han contribuido notablemente a la mejora del artículo. No obstante, las opiniones expresadas son de exclusiva responsabilidad de las personas autoras del artículo.

2 El recorte de la población ocupada se realiza a partir de dos cuestiones: por un lado, las definiciones de actividad declaradas para la unidad económica a raíz de las definiciones del Observatorio de Empleo y Dinámica Empresarial del Ministerio de Trabajo, Empleo y Seguridad Social; por otro, tipo de actividad principal dentro de dicha unidad declarada por parte de la persona encuestada ante las EPH. Si bien a nivel de demanda de fuerza de trabajo el sector vinculado a la compra y venta de bienes automotores figura en el un sector asociado al "comercio", la definición del colectivo de trabajo lo ubica como parte de la cadena de valor de la industria automotriz, siendo para el caso que el Convenio Colectivo de Trabajo suscribe las firmas de la Asociación de Concesionarios de Automotores de la República Argentina (ACARA) y el Sindicato de Mecánicos y Afines del Transporte Automotor de la República Argentina (SMATA).

3 Los detalles se encuentran en las notas metodológicas correspondientes al Boletín de empresas -serie anual 2016- y al Boletín de empleo registrado -serie anual 2017-.

4 En este sentido, numerosos debates han planteado que las dificultadas en el análisis del mercado de trabajo se presentan en gran medida en la fiabilidad y disponibilidad de las series continuas de las bases de datos y la comparación interperíodos en base a los diseños muestrales utilizados (Wlosko, et al., 2016, p. 8). Teniendo estas cuestiones presentes, los datos presentados deben ser considerados en tanto estimaciones y tendencias. 
5 La misma es a partir de la cotización de la divisa para el 29 de diciembre de los años correspondientes. Dichos valores son: 20061 usd= 3,06 pesos argentinos; 20111 usd= 4,29 pesos argentinos; 20161 usd= 15,70 pesos argentinos. El registro del valor de la divisa se encuentra disponible en el siguiente enlace: https://www.bna.com.ar/Personas. 